\title{
Incorporating Lung Ultrasound in Clinical Pulmonary Infection Score as an Added Tool for Diagnosing Ventilator- associated Pneumonia: A Prospective Observational Study from a Tertiary Care Center
}

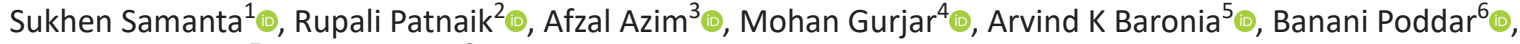 \\ Ratender K Singh ${ }^{7}{ }^{\circ}$ Zafar Neyaz $^{8}{ }^{\circ}$
}

\begin{abstract}
Background: Clinical pulmonary infection score (CPIS) is an established diagnostic parameter for ventilator-associated pneumonia (VAP). Lung ultrasound (LUS) is an evolving tool for diagnosing VAP. Various scores have been proposed for the diagnosis of VAP, taking LUS as a parameter. We proposed whether replacing LUS with chest radiograph in CPIS criteria will add to the diagnosis of VAP. The current study was done to evaluate the diagnostic accuracy of LUS alone and in combination with clinical and microbiological criteria for VAP by replacing chest radiograph with LUS in CPIS.

Materials and methods: We conducted a prospective single-center observational study including 110 patients with suspected VAP to investigate the diagnostic accuracy of LUS. Quantitative mini-bronchoalveolar lavage (mini-BAL) culture was considered the gold standard for diagnosis of VAP. Here, the authors have explored the combination of LUS, clinical, and microbiology parameters for diagnosing VAP. On replacing chest radiograph with LUS, sono-pulmonary infection score (SPIS) and modified SPIS (SPIS-mic, SPIS-cult) was formulated as a substitute for CPIS.

Results: Overall LUS performance for VAP diagnosis was good with sensitivity, specificity, positive or negative predictive value, and positive or negative likelihood ratios of $91.3 \%, 70 \%, 89 \%, 75 \%, 3$, and 0.1 , respectively. Adding microbiology culture to LUS increased diagnostic accuracy. The area under the curve for SPIS and modified SPIS were $0.808,0.815$, and 0.913 , respectively.

Conclusions: The diagnosis of VAP requires agreement between clinical, microbiological, and radiological criteria. Replacing chest radiograph with LUS in CPIS criteria (SPIS) increases diagnostic accuracy for VAP. Adding clinical and culture data to SPIS provided the highest diagnostic accuracy. Clinical parameters along with lung ultrasound increase diagnostic accuracy for VAP.

Keywords: Air bronchogram, Clinical pulmonary infection score, Lung ultrasound, Mini-bronchoalveolar lavage, Ventilator-associated pneumonia.

Indian Journal of Critical Care Medicine (2021): 10.5005/jp-journals-10071-23759
\end{abstract}

\section{INTRODUCTION}

Ventilator-associated pneumonia (VAP) is the commonest nosocomial infections in mechanically-ventilated patients. ${ }^{1}$ It can be easily understood that VAP is associated with increased mortality, morbidity, and extra healthcare costs. ${ }^{2}$ Appropriate antibiotic therapy in patients with VAP considerably improves outcomes, making rapid identification of such patients an important clinical goal. The clinical pulmonary infection score (CPIS) (without using microbiologic data) or a modified CPIS (using microbiological data; e.g., CPIS-mic, CPIS-cult) have also been proposed to improve diagnostic accuracy. A CPIS $\geq 6$ is often regarded as consistent with a diagnosis of pneumonia. ${ }^{3}$ Ultrasound machine is the new stethoscope and its use has been extended to various fields including bedside diagnosis of pneumothorax, atelectasis, pleural effusion, and consolidation in mechanically ventilated patients. ${ }^{4,5}$ At the bedside, it has the potential to replace the routine chest radiographs (CXR). In a retrospective study, a score combining procalcitonin (PCT) report, along with LUS consolidation, performed better than CPIS. ${ }^{6}$ Recently, in a prospective multi-centric study,
1Departments of Critical Care, Orchid Medical Centre, Ranchi, Jharkhand, India

${ }^{2-7}$ Departments of Critical Care Medicine, Sanjay Gandhi Postgraduate Institute of Medical Sciences, Lucknow, Uttar Pradesh, India

${ }^{8}$ Department of Radiodiagnosis, Sanjay Gandhi Postgraduate Institute of Medical Sciences, Lucknow, Uttar Pradesh, India

Corresponding Author: Afzal Azim, Departments of Critical Care Medicine, Sanjay Gandhi Postgraduate Institute of Medical Sciences, Lucknow, Uttar Pradesh, India, Phone: +91 8004904730, e-mail: draazim2002@gmail.com

How to cite this article: Samanta S, Patnaik R, Azim A, Gurjar M, Baronia AK, Poddar B, et al. Incorporating Lung Ultrasound in Clinical Pulmonary Infection Score as an Added Tool for Diagnosing Ventilator-associated Pneumonia: A Prospective Observational Study from a Tertiary Care Center. Indian J Crit Care Med 2021;25(3): 284-291.

Source of support: Nil

Conflict of interest: None

(c) Jaypee Brothers Medical Publishers. 2021 Open Access This article is distributed under the terms of the Creative Commons Attribution 4.0 International License (https://creativecommons.org/licenses/by-nc/4.0/), which permits unrestricted use, distribution, and non-commercial reproduction in any medium, provided you give appropriate credit to the original author(s) and the source, provide a link to the Creative Commons license, and indicate if changes were made. The Creative Commons Public Domain Dedication waiver (http://creativecommons.org/publicdomain/zero/1.0/) applies to the data made available in this article, unless otherwise stated. 
the authors have proposed a score-based diagnostic approach in VAP using LUS, purulent secretions, and endotracheal aspirate microscopy. ${ }^{7}$ Lack of uniformity between the CPIS and these new scores has led to ambiguity amongst the clinicians. We hypothesis that replacing LUS with chest radiograph in CPIS criteria will improve the diagnosis of VAP.

\section{Materials and Methods \\ Patient Selection and Study Design}

The current observational study was prospectively conducted over 18 months in a 20-bedded intensive care unit of a teaching hospital located in North India. Approval from the ethics committee of the institute was taken as per protocol. A total of 115 patients with suspected clinical VAP were included in this study. Clinical suspicion of VAP was done when the flowing criteria were met: at least 48 hours of mechanical ventilation, CXR suggestive of new or increased infiltrates, and at least two of the given four clinical criteria: temperature $\geq 38.5^{\circ} \mathrm{C}\left(101{ }^{\circ} \mathrm{F}\right)$ or $<36{ }^{\circ} \mathrm{C}\left(97^{\circ} \mathrm{F}\right)$, total leukocyte count $>10000 / \mu \mathrm{L}$ or $<4,000 / \mu \mathrm{L}$, the partial pressure of oxygen in arterial blood or inspired oxygen fraction (P/F) ratio $<300$, and purulent respiratory secretions. VAP diagnosis was confirmed by positive results of mini-BAL quantitative culture ( $\geq 10^{4} \mathrm{CFU} / \mathrm{mL}$ ). Patients who did not meet the above criteria or were already diagnosed with pneumonia and had any contraindication to mini-BAL were excluded from the study. Of the 115 recruited patients, five patients were later excluded due to incomplete data. Informed written consent was obtained from close relatives in all cases for LUS, respiratory sample collection, and the study.

\section{USG Technique}

Lung ultrasound was performed using a portable ultrasound unit (Micro MaXXSonosite, Gurgaon, India) with a $3.5 \mathrm{MHz}$ rounded transducer within 12 hours of the point at which criteria were met for suspected VAP. It was performed by a consultant radiologist experienced in performing the same at least 250 times.

Lung USG was done to examine areas of abnormality by dividing each hemithorax into three parts with the help of anterior and posterior axillary lines. A horizontal line further divided these parts into upper and lower quadrants, thus making six quadrants in each hemithorax. Lung USG findings were reported as A-lines (normal lung), B-lines (coalescent and non-coalescent), pleural effusions, and consolidation (lobar, subpleural, and areas of dynamic air bronchograms). The presence of dynamic air bronchogram within areas of consolidation (inhomogeneous or tissue-like echotexture), lobar or subpleural consolidation were considered diagnostic of VAP on sonography (Figs 1-3).

Clinical pulmonary infection score (CPIS) and modified CPIS (CPIS-mic; CPIS with positive microscopy of tracheal aspirate and CPIS-cult; CPIS with a positive tracheal aspirate culture) are well-accepted scores for diagnosing VAP in ICU settings. In this study, LUS-based score was computed similar to CPIS, where we replaced chest X-ray with LUS findings. We coined the score as sono-pulmonary infection score (SPIS). First is the SPIS, where we incorporated LUS finding instead of CXR finding of CPIS. We assigned two points for $\geq$ one area of dynamic air bronchogram. One point was given for $\geq$ two sub-pleural consolidation or lobar consolidation areas or $\geq$ one sub-pleural, and $\geq$ one lobar consolidation. Other parameters in SPIS were similar to CPIS. Similar to modified CPIS, modified SPIS was also formulated. We needed positive microscopy of tracheal aspirate and added one point to

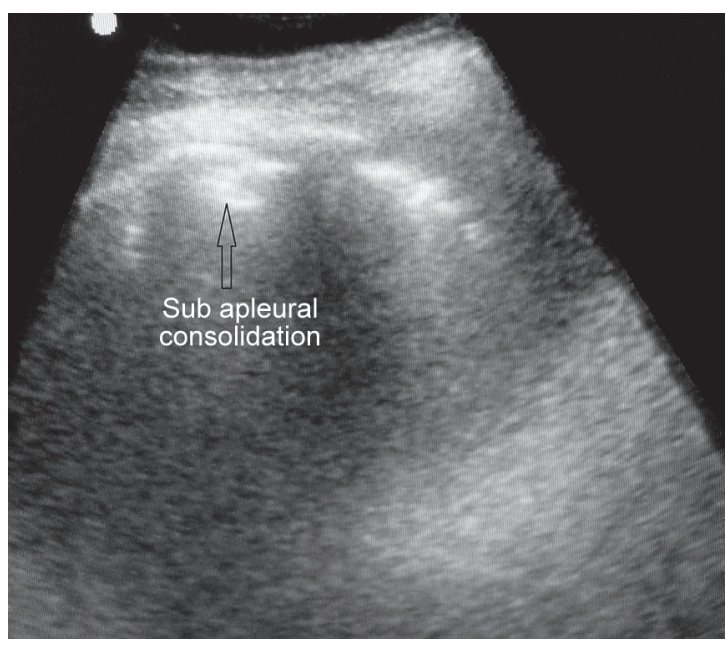

Fig. 1: Lung ultrasound showing sub-pleural consolidation

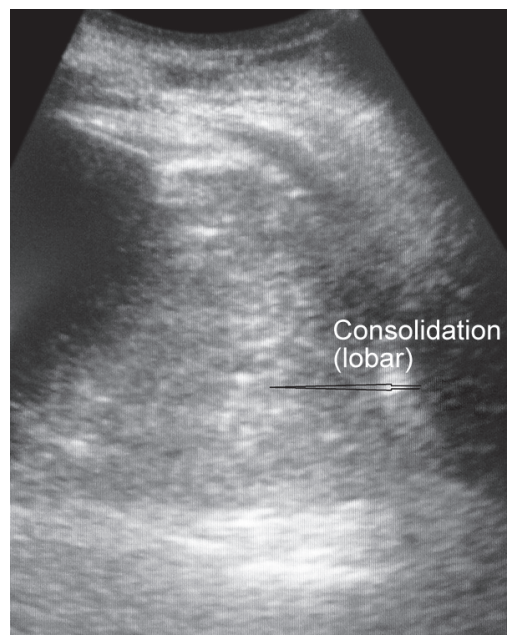

Fig. 2: Lung ultrasound demonstrated lobar consolidation

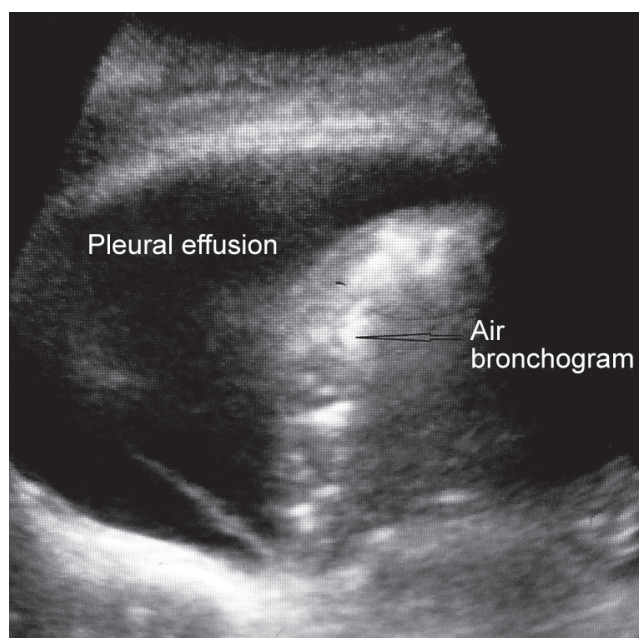

Fig. 3: Lung ultrasound highlighting air bronchogram within lobar consolidation along with pleural effusion 
Table 1: Description of parameters of scores used in the study

\begin{tabular}{|c|c|}
\hline SPIS (sono-pulmonary infection score) & Points \\
\hline \multicolumn{2}{|l|}{ Lung ultrasound (LUS) } \\
\hline $\begin{array}{l}\text { No consolidation and dynamic air bronchogram on lung } \\
\text { ultrasound }\end{array}$ & 0 \\
\hline $\begin{array}{l}\geq 2 \text { area with sub-pleural consolidation or lobar } \\
\text { consolidation or } \geq 1 \text { subpleural and } \geq 1 \text { lobar consolidation }\end{array}$ & 1 \\
\hline$\geq 1$ area with dynamic air bronchogram & 2 \\
\hline \multicolumn{2}{|l|}{ Purulent secretion } \\
\hline Absent or minimal & 0 \\
\hline Non-purulent & 1 \\
\hline Purulent & 2 \\
\hline \multicolumn{2}{|l|}{ Endotracheal aspirate microscopy/culture } \\
\hline Negative microscopy/culture & 0 \\
\hline Positive microscopy & 1 \\
\hline Positive culture(same organism) & 2 \\
\hline \multicolumn{2}{|l|}{ Temperature } \\
\hline$\leq 38.4$ and $\geq 36.5$ & 0 \\
\hline$\geq 38.5$ & 1 \\
\hline$\geq 39$ or $\leq 36$ & 2 \\
\hline \multicolumn{2}{|l|}{ Leukocyte count/ $\mu \mathrm{L}$} \\
\hline$\geq 4000$ and $\leq 11000$ & 0 \\
\hline$<4000$ and $>11000$ & 1 \\
\hline \multicolumn{2}{|l|}{ Oxygenation P/F } \\
\hline$>240$ or presence of ARDS & 0 \\
\hline$\leq 240$ or absence of ARDS & 2 \\
\hline
\end{tabular}

CPIS (clinical pulmonary infection score)

\begin{tabular}{ll}
\hline Temperature & \\
$\leq 38.4$ and $\geq 36.5$ & 0 \\
$\geq 38.5$ & 1 \\
$\geq 39$ or $\leq 36$ & 2 \\
Leukocyte count/ $\mathrm{LL}$ & \\
$\geq 4000$ and $\leq 11000$ & 1 \\
$<4000$ and $>11000$ & \\
Oxygenation & 0 \\
$>240$ or presence of ARDS & 2 \\
$\leq 240$ or absence of ARDS & \\
Tracheal secretion & 0 \\
Absent or minimal & 1 \\
Non-purulent & 2 \\
Purulent & \\
Chest radiograph & 0 \\
No infiltrate & 1 \\
Patchy infiltrate & 2 \\
Localized infiltrate & \\
Endotracheal aspirate microscopy/culture & \\
Negative microscopy/culture & \\
Positive microscopy & \\
Positive culture (same organism) & 1
\end{tabular}

Notes: ARDS, acute respiratory distress syndrome; positive microscopy result means positive result on direct gram stain examination of endotracheal aspirate. Positive culture means positive result on the culture of endotracheal aspirate with colony-forming unit $\geq 10^{5}$

SPIS and called as SPIS-mic. The culture report of tracheal aspirate was added, and two points were given above SPIS, and it was called SPIS-cult (Table 1).

\section{Data Collection}

Demographic characteristics and ICU prognostication scores, that is, acute physiologic and chronic health evaluation (APACHE) II score and sequential organ dysfunction assessment (SOFA), were recorded at admission. Clinical suspicion of VAP was taken as a trigger for the collection of mini-BAL and endotracheal aspirate samples for gram stain and culture. At inclusion (clinical suspicion of VAP), CXR reports were evaluated, LUS was done, respiratory samples (both tracheal aspirate and mini-BAL) were collected by the resident doctor, and respiratory therapist with standardized asepsis technique. Variables for CPIS and SPIS were collected, and PCT was sent. For CPIS-mic, CPIS-cult, SPIS-mic, SPIS-cult, microbiological data of tracheal aspirate samples were taken into account. At the time of patient inclusion, SOFA was recalculated. All these were performed within 12 hours of clinical suspicion of VAP. We considered a guideline-based diagnostic threshold of $\geq 104 \mathrm{CFU} / \mathrm{mL}$ of a mini-BAL sample as indicative of bacterial pneumonia and was taken as a confirmed diagnosis of VAP. The number of mechanical ventilator days, length of ICU, and hospital stay were calculated, and at the patient's discharge or death from ICU, we documented them as survivors or non-survivors.

\section{Mini-bronchoalveolar lavage}

Mini-bronchoalveolar lavage (mini-BAL) specimens were collected by trained residents or respiratory therapists in conjunction with a "catheter in catheter" technique with sterile normal saline. The standard volume of saline instilled in mini-BAL procedures was $20 \mathrm{~mL}$. Initial $10 \mathrm{~mL}$ were routinely discarded. The final segmental placement of the mini-BAL catheter was blind. Samples were studied for Gram stain and microbiological culture. Microscopy report was usually available within 4-6 hours of sample dispatch. In this study, we considered mini-BAL culture positive as the gold standard for the diagnosis of VAP. We took mini-BAL culture as the gold standard for VAP to avoid commensals from upper respiratory flora and avoid contamination. European guidelines still prefer quantitative culture diagnosed by mini-BAL culture as the gold standard for VAP diagnosis.

\section{Statistical Analysis}

We included all possible suspected VAP cases during the study period. Descriptive data were summarized as mean (SD), median (interquartile range) for non-normal data, or number (\%). Two groups (mini-BAL culture-positive VAP and non-VAP) were compared by using Mann-Whitney test for numerical data and Fischer's exact test for categorical data. Sensitivity, specificity, positive or negative predictive value, and positive or negative likelihood ratios were calculated for CPIS, SPIS, LUS parameters, CXR, and serum PCT. Results were expressed with 95\% confidence intervals (Cls). Receiver operating curves (ROC) were plotted with the area under the curve for CPIS, CPIS-mic, CPIS-cult, SPIS, SPISmic, and SPIC-cult.

\section{Results}

A total of 386 patients got admitted during the study period. One hundred fifteen patients were initially enrolled for the study after fulfilling the inclusion criteria. Data were analyzed for 110 patients due to inadequate lung ultrasound $(n=2)$ and microbiology culture findings $(n=3)$. Of the 110 patients, 80 patients had mini-BAL culture-confirmed VAP that was considered the gold standard for VAP diagnosis. The flowchart of patient distribution and outcome is summarized in Fig. 4.

Demographic data, that is, age, sex, type of patients (medical or surgical), co-morbidities, and previous hospital stay before 


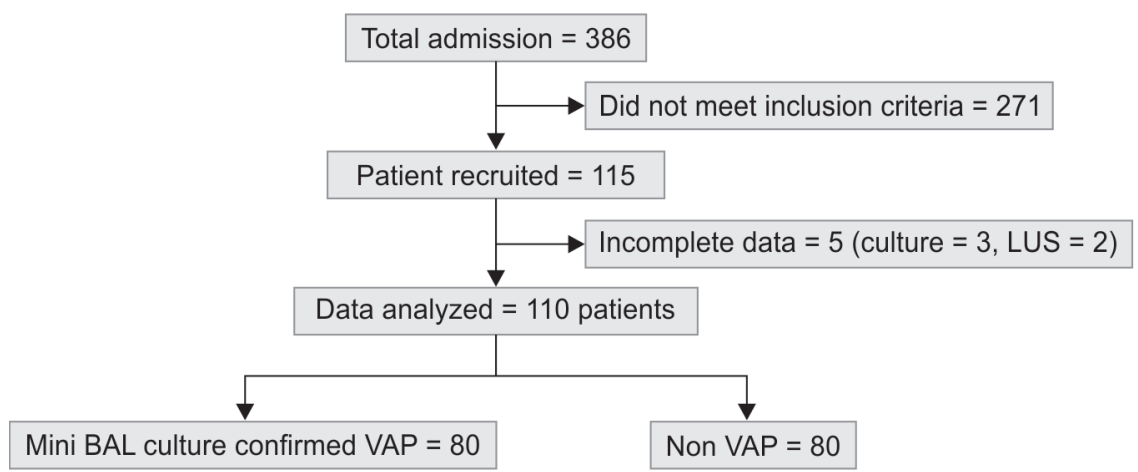

Fig. 4: Flow chart showing patient screening, study inclusion, and outcome

Table 2: Patient characteristics and outcome of included patients

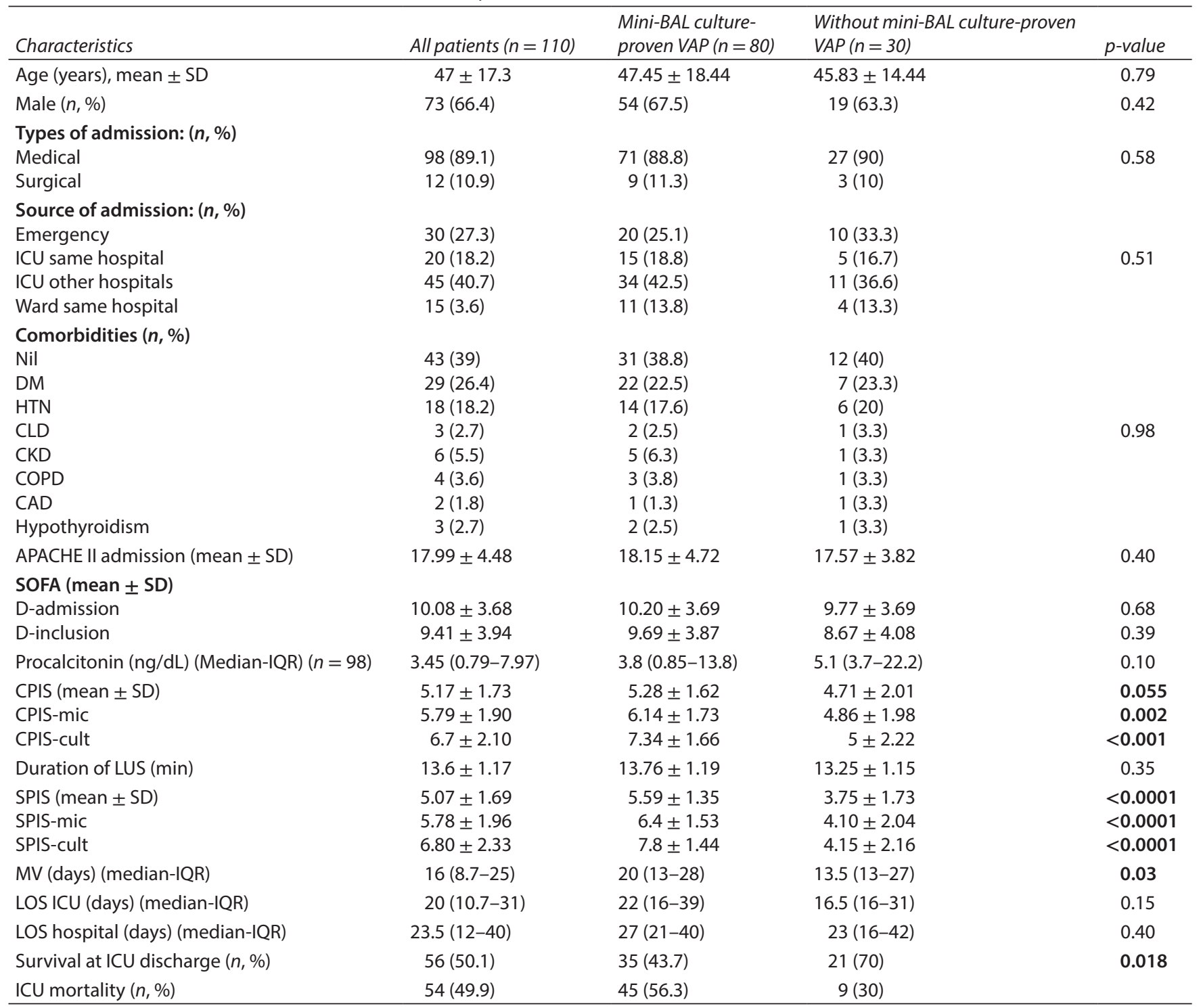

Notes: APACHE II, acute physiology chronic health evaluation; CAD, coronary artery disease; CKD, chronic kidney disease; CLD, chronic liver disease; COPD, chronic obstructive pulmonary disease; CPIS, clinical pulmonary infection score; DM, diabetes mellitus; HTN, hypertension; ICU, intensive care unit; LOS, length of stay; MV, mechanical ventilation SOFA, sequential organ failure assessment; SPIS, sono-pulmonary infection score; VAP, ventilator-associated pneumonia 
admission (other hospital ICU, same hospital other ICU or ward, and emergency department) are represented in Table 2. APACHE II at admission was 18 in mini-BAL culture-confirmed VAP. SOFA at inclusion was 9 in mini-BAL culture-confirmed VAP. Patients with positive mini-BAL culture remained significantly more days on mechanical ventilation than culture-negative (20 days vs 13 days, $p=0.03$ ) and had more ICU mortality (56 vs $30 \%, p=0.018$ ). Gram-negative organisms (88.7\%) were the predominant finding in culture growth, with Acinetobacter baumannii being the most common organism, followed by Pseudomonas aeruginosa (Table 3).

Diagnostic accuracy of CXR, LUS, including individual findings, and procalcitonin are described in Table 4. CXR 2 (localized infiltrate on chest X-ray) had increased specificity and positive predictive value to diagnose true VAP (mini-BAL culture-confirmed)

Table 3: Microbiological profile of sample from mini-BAL culture $(n=80)$

\begin{tabular}{ll}
\hline Organism on positive mini-BAL fluid & Days of study No (\%) \\
\hline Gram-negative organism & $71(88.7 \%)$ \\
Acinetobacter baumannii & $36(45 \%)$ \\
Pseudomonas aeruginosa & $17(21.5 \%)$ \\
Klebsiella pneumoniae & $12(15 \%)$ \\
Escherichia coli & $2(2.5 \%)$ \\
Stenotrophomonas & $2(2.5 \%)$ \\
Others & $2(2.5 \%)$ \\
Gram-positive organism & $5(6.3 \%)$ \\
Staphylococcus aureus & $4(5 \%)$ \\
Others & $1(1.3 \%)$ \\
Fungus & $4(5 \%)$ \\
\hline
\end{tabular}

Notes: Seven mini-BAL specimens (8.2\%) were positive for multiple organisms than diffuse infiltrate (CXR 1). LUS 2 ( $\geq 1$ area with dynamic air bronchogram) had the highest specificity and positive predictive value to diagnose VAP. Overall LUS performance for diagnosis of VAP (microbiologically confirmed) is good with sensitivity, specificity, positive or negative predictive value, and positive or negative likelihood ratios of $91.3 \%$ (82.8-96.4), 70\% (50.6-85.3), 89\% (80.2-94.9), 75\% (55.1-89.3), 3 (1.8-5.3), 0.1 (0.1-0.3), respectively. Serum PCT values $\geq 1 \mathrm{ng} / \mathrm{dL}$ was found to be a poor predictor of VAP.

Diagnostic performances of scores combining clinical, microbiological, and radiological data are described in Table 5. To diagnose mini-BAL culture-confirmed VAP, CPIS $\geq 6$ had a positive predictive value of $79.3 \%$. CPIS with positive microscopy or culture of endotracheal aspirate had an increased positive predictive rate for definite diagnosis of VAP. SPIS $\geq 6$ had an $82.2 \%$ positive predictive rate for diagnosis of VAP. The addition of microbiology culture of tracheal aspirate along with LUS improved diagnostic accuracy. SPIS-cult (SPIS with a positive culture of endotracheal aspirate) had the highest specificity and positive predictive value (90.9\%) (Table 4).

Receiver operating characteristic curves (ROC) with areas under the curve (AUC) for CPIS, CPIS-mic, CPIS-cult; SPIS, SPIS-mic, SPIScult for diagnosis of VAP, are described in Table 6 and Figure 5. SPIS alone had almost equivalent AUC as of CPIS-cult (0.80). Amongst the combined score, SPIS-cult AUC yield was the highest (0.91).

\section{Discussion}

Lung ultrasound is an evolving added tool for the diagnosis of VAP. For early diagnosis of VAP, various scoring systems have been proposed, like CEPPIS (chest echography and procalcitonin pulmonary infection score) and VPLUS (ventilator-associated pneumonia lung ultrasound), which incorporated lung ultrasound findings along with the clinical and microbiological culture of

Table 4: Performance of CXR, LUS, and procalcitonin at inclusion for diagnosis of VAP (mini-BAL culture-confirmed)

\begin{tabular}{|c|c|c|c|c|c|c|}
\hline Parameters & Sensitivity & Specificity & $P P V$ & NPV & $L R+$ & $L R-$ \\
\hline CXR 1 & $72.5(61.4-81.9)$ & $26.7(12.3-45.9)$ & $72.5(61.4-81.9)$ & $26.7(12.3-45.9)$ & $0.9(0.8-1.3)$ & $1.03(0.5-2.1)$ \\
\hline CXR 2 & $27.5(18.1-38.6)$ & $73.3(54.1-87.7)$ & $73.3(54.1-87.7)$ & $27.5(18.1-38.6)$ & $1.03(0.5-2.1)$ & $0.9(0.8-1.3)$ \\
\hline LUS1 & $87.1(78-93.4)$ & $56(34.9-75.6)$ & $87.1(78-93.4)$ & $56(34.9-75.6)$ & $1.9(1.3-3.1)$ & $0.2(0.1-0.4)$ \\
\hline LUS 2 & $42.8(31.6-54.7)$ & $91.7(61.5-99.8)$ & $97.1(84.7-99.9)$ & $20(10.4-32.9)$ & $5.1(0.8-34.2)$ & $0.62(0.5-0.8)$ \\
\hline LUS overall & $91.3(82.8-96.4)$ & $70(50.6-85.3)$ & $89(80.2-94.9)$ & $75(55.1-89.3)$ & $3(1.8-5.3)$ & $0.1(0.1-0.3)$ \\
\hline Procalcitonin $\geq 1 \mathrm{ng} / \mathrm{dL}(n=98)$ & $59.7(47.5-71.1)$ & $15.4(4.4-37.9)$ & $66.2(53.4-77.4)$ & $12.1(3.4-28.2)$ & $0.7(0.6-0.9)$ & $2.6(1-6.7)$ \\
\hline
\end{tabular}

Notes: Data are presented as \% (95\% CI). CXR, chest X-ray; CXR 1, diffuse infiltrate; CXR 2, localized infiltrate; LR+, positive likelihood ratio; LR-, negative likelihood ratio; LUS, lung ultrasound, LUS1, LUS 2 (Table 1 for detail); NPV, negative value; PPV, positive predictive value; VAP, ventilator-associated pneumonia

Table 5: Performance of CPIS, CPIS-mic, CPIS-cult, SPIS, SPIS-mic, and SPIS-cult at inclusion for diagnosis of VAP (mini-BAL culture-confirmed)

\begin{tabular}{lllllll}
\hline Parameters & Sensitivity\% & \multicolumn{1}{l}{ Specificity\% } & PPV\% & \multicolumn{1}{l}{ NPV\% } & \multicolumn{1}{l}{$L$ +} & \multicolumn{1}{l}{ LR- } \\
\hline CPIS $\geq 6$ & $57.5(45.9-68.5)$ & $60(40.6-77.3)$ & $79.3(66.6-88.8)$ & $34.6(21.9-49.1)$ & $1.43(0.89-2.32)$ & $0.71(0.48-1.04)$ \\
CIPS mic $\geq 6$ & $71.3(60.1-80.8)$ & $60(40.6-77.3)$ & $82.6(71.7-90.7)$ & $43.9(28.5-60.3)$ & $1.70(1.1-2.8)$ & $0.48(0.3-0.8)$ \\
CPIS cult $\geq 6$ & $78.8(68.2-87.1)$ & $63.3(43.9-80.1)$ & $85.1(74.9-92.3)$ & $53(35.5-69.6)$ & $2.15(1.3-3.5)$ & $0.34(0.2-0.6)$ \\
SPIS $\geq 6$ & $46.3(35-57.8)$ & $73.3(54.1-87.7)$ & $82.2(67.9-92)$ & $33.9(22.6-46.7)$ & $1.37(0.9-3.3)$ & $0.73(0.5-0.9)$ \\
SPIS mic $\geq 6$ & $77.5(66.8-86.1)$ & $76.7(57.7-90.1)$ & $89.9(80.2-95.8)$ & $56.1(39.8-71.5)$ & $3.32(1.7-6.4)$ & $0.3(0.2-0.5)$ \\
SPIS cult $\geq 6$ & $87.5(78.2-93.8)$ & $76.7(57.7-90.1)$ & $90.9(82.2-96.3)$ & $69.7(51.3-84.4)$ & $3.8(1.9-7.2)$ & $0.2(0.1-0.3)$ \\
\hline
\end{tabular}

Notes: Data are presented as \% $(95 \% \mathrm{Cl})$. CPIS, clinical pulmonary infection score; LR+, positive likelihood ratio, LR-, negative likelihood ratio; LUS, lung ultrasound; NPV, negative value; PPV, positive predictive value; SPIS, sono-pulmonary infection score 


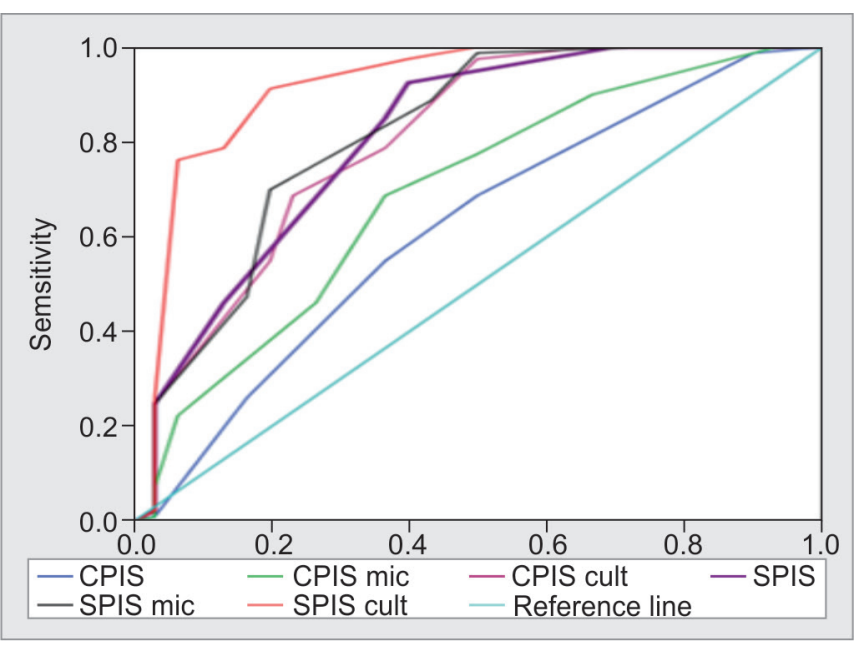

Fig. 5: Receiver operating characteristic (ROC) curves of CPIS, CPIC-mic, CPIS-cult, SPIS, SPIS-mic, and SPIS-cult

Table 6: Receiver operating characteristic (ROC) AUCs of predictors for VAP diagnosis

\begin{tabular}{llllc}
\hline Predictors & AUC & SE & $95 \% \mathrm{Cl}$ & $p$-value \\
\hline CPIS & 0.617 & 0.062 & $0.495-0.739$ & 0.06 \\
CPIS-mic & 0.689 & 0.059 & $0.573-0.804$ & 0.002 \\
CPIS-cult & 0.801 & 0.052 & $0.699-0.903$ & $<0.0001$ \\
SPIS & 0.808 & 0.051 & $0.707-0.908$ & $<0.0001$ \\
SPIS-mic & 0.815 & 0.051 & $0.714-0.915$ & $<0.0001$ \\
SPIS-cult & 0.913 & 0.038 & $0.839-0.988$ & $<0.0001$ \\
\hline
\end{tabular}

Notes: AUC, area under curve; CPIS, clinical pulmonary infection score; CPIS mic, clinical pulmonary infection score with gram stain; CPIS cult, clinical pulmonary infection score with culture; ROC, receiver operating characteristic curve; SE, standard error; SPIS, sono-pulmonary infection score; SPIS mic, sono-pulmonary infection score with gram stain; SPIS cult, sono-pulmonary infection score with culture

endotracheal aspirate for early diagnosis of VAP. CPIS score is the most widely used clinic-microbiological score, which considers chest radiograph findings as to the radiological criteria. Scores like CEPPIS and VPLUS take into account LUS findings, though they are of immense help but challenging to remember at bedside and practice. We evaluated the feasibility of incorporating lung ultrasound findings into the CPIS score and formulated the SPIS score, where we used lung ultrasound instead of the chest radiograph.

CPIS was first defined by Pugin et al. and demonstrated that it had a high sensitivity (93\%) and specificity (100\%). ${ }^{8}$ Later on, Croce et al. evaluated CPIS and confirmed its sensitivity as $61 \%$ and specificity as $43 \% .{ }^{9}$ Schurink et al. found sensitivity as $83 \%$ and specificity as $17 \%$ using six parameters (incorporating culturemodified CPIS), taking a threshold value of $>6$ for the evaluation of CPIS. ${ }^{10}$ Further, studies comparing CPIS with a histological diagnosis and BAL fluid-established diagnosis of VAP showed a lower diagnostic performance compared with the original research, with limited sensitivity and specificity. ${ }^{11,12}$ In our study, we found sensitivity, specificity, PPV, NPV, and positive or negative likelihood ratios of $57.5 \%(45.9-68.5), 60 \%(40.6-77.3), 79.3 \%(66.6-88.8)$, $34.6 \%$ (21.9-49.1), $1.43(0.89-2.32), 0.71$ (0.48-1.04) (Table 5), respectively with ROC area under the curve of 0.617 for a CPIS > 6 (Table 6). Microscopy and culture are incorporated in CPIS and is commonly known as modified CPIS. Singh et al. used the CPIS as a screening tool for decision-making regarding antibiotic therapy, incorporating the Gram stain result. ${ }^{13}$ In our study, we noted that adding microscopy (Gram stain) to CPIS did not improve any diagnostic performance (CPIS AUC is 0.617 and CPIS-mic AUC is 0.689 ) but adding quantitative culture report improved accuracy of diagnosis by increasing ROC area under the curve from 0.617 to 0.801 . Serum PCT level in suspected VAP can help improve diagnosis, but as a single parameter, it is not reliable.

In the formulated SPIS score for the diagnosis of VAP, all other parameters remaining the same as CPIS, we used lung ultrasound instead of the chest radiograph. Studies have proven a limited diagnostic performance of bedside portable CXR in critically ill patients. ${ }^{14}$ In this study, we found CXR showing diffuse infiltration has $72.5 \%$ (61.4-81.9), 26.7\% (12.3-45.9) sensitivity and specificity, respectively. Specificity improved in case of localized infiltration but at the cost of low sensitivity (Table 4). Diagnosis of VAP by computed tomography (CT) scan was carried out by Winer-Muram et al. on ARDS patients. They found diagnostic accuracy improved with the use of CT scans. ${ }^{15}$ Limitations of CT scan, such as less readily accessible, radiation hazards, needs transportation, and does not give any specific findings of VAP except consolidation and air bronchogram. To evaluate new infiltration, we need a baseline CT scan. ${ }^{14}$ This evaluation of baseline information and new infiltration can be easily performed by LUS at the bedside. ${ }^{16}$ Meta-analysis supports the fact that LUS can diagnose community-acquired pneumonia in the emergency department. ${ }^{17}$ For ICU patient's bedside, LUS is frequently used to detect pleural effusion, lung consolidation, and AIS with good sensitivity and specificity. The higher rate of detection from LUS, combined with its ease of use and increasing accessibility, makes it a strong point of care diagnostic tool. A combination of non-aerated lung tissues and dynamic air bronchogram is the most characteristic sonographic feature of inflammatory lung consolidation. Our study found sensitivity and specificity of LUS as $91.3 \%(82.8-96.4)$ and $70 \%$ (50.6-85.3), respectively, and is comparable to other studies. ${ }^{18-21}$ LUS 2 ( $\geq 1$ area with dynamic air bronchogram) had the highest specificity and positive predictive value to diagnose VAP (Table 4).

The diagnosis of VAP requires agreement between clinical, microbiological, and radiological criteria. ${ }^{22}$ In a retrospective study by Zagli et al., total leucocyte count was replaced by PCT. They formulated a scale CEPPIS incorporating tracheal secretion, $P C T$, the culture of tracheal secretion, LUS consolidation, and oxygenation as different variables. ${ }^{6}$ They found a CEPPIS > five was significantly better in predicting VAP (OR, 23.78; sensitivity, $80.5 \%$; specificity, $85.2 \%$, and AUC 0.829) than a CPIS $>6$. Serum PCT level in suspected VAP can help improve diagnosis, but it is not reliable as a single parameter. In our study, serum PCT was a poor indicator of VAP with sensitivity and specificity of $59.7 \%$ (47.5-71.1) and $15.4 \%$ (4.4-37), respectively. Mongodi et al. published a score-based protocol ventilator-associated pneumonia lung ultrasound (VPLUS) and VPLUS with positive gram smear (VPLUS-gram) for early diagnosis of VAP using ultrasound finding, microscopy by Gram stain along with purulent tracheal aspirate. ${ }^{7}$ Based on VPLUS-gram, they recommend early initiation of antibiotics. Zagli et al. performed lung ultrasound in the nonspecific period between the third to the fifth day of ICU stay with the diagnosis based on subpleural echo poor regions or tissue-like structures. ${ }^{6}$ Mongodi et al. have nicely described the ultrasound scoring system. ${ }^{7}$ They have given utmost importance to 
Gram stain examination and early initiation of antibiotics. However, in their clinico-radiological and microbiological VPLUS and VPLUSgram scores, purulent secretion was assessed as the only clinical parameter that is subjective.

Our present study scoring system SPIS was formulated using all the variables of CPIS, including lung ultrasound findings. Microscopy by Gram stain and culture reports were used in modified SPIS (SPIS-mic and SPIS-cult). SPIS $\geq 6$ had an $82.2 \%$ positive predictive rate for diagnosis of VAP. The addition of microbiology culture of tracheal aspirate along with LUS improved diagnostic accuracy. SPIS-cult (SPIS with a positive culture of endotracheal aspirate) had the highest specificity and positive predictive value (90.9\%) (Table 4). Our result suggests the best AUROC for SPIS-cult (0.913).

We can start appropriate empirical antibiotics based on clinical parameters and LUS, which generally takes 15 minutes after obtaining the respiratory sample. Taking a sample from the respiratory tract and getting a Gram stain report generally takes 3-6 hours. Our study showed the prevalence of gram-negative organisms (88.7\%). It may be clinically justified not to wait for microscopy but to start early antibiotics in patients with suspected VAP, evident by clinical and LUS findings. The Clinical and Laboratory Standards Institute (CLSI) recommends establishing a unitspecific antibiogram. ${ }^{23}$ This is of utmost importance, as bacterial susceptibilities to antibiotics are subject to geographical variation, which was recently highlighted by Becher et al. ${ }^{24}$ Novel methods of rapid pathogen identification and their sensitivity testing may enhance the likelihood of administering timely and appropriate empirical antibiotic therapy.

Ours is the first study that evaluated the utility of lung ultrasound for the diagnosis of VAP, incorporating LUS in the existing CPIS score. Scores like CEPPIS and VPLUS proposed previously have evaluated lung ultrasound's utility as a tool for early VAP diagnosis. SPIS, a modification of CPIS, can be of utility as a tool for accurate VAP diagnosis, with advantages of easy to practice at the bedside and no ambiguity amongst clinicians.

\section{Conclusions}

Clinical, ultrasound, and microbiology-based scoring systems are better than clinical decision-making for the early diagnosis of VAP. A multi-centric randomized trial with large sample size is warranted to compare LUS-based scoring (SPIS and modified SPIS) with clinicalbased scoring (CPIS and modified CPIS).

\section{LIMITATIONS}

The significant limitations of our study are that it is a single-center observational study. Large numbers of patients with mixed population might provide better analysis. We did not perform daily lung ultrasound. We considered quantitative mini-BAL culture as a gold standard test, but nothing has yet been established as a gold standard.

\section{Abbreviations}

\section{BAL: Bronchoalveolar lavage}

CPIS: Clinical pulmonary infection score

CPIS-mic: Clinical pulmonary infection score-microscopy

CPIS-cult: Clinical pulmonary infection score-culture

LUS: Lung ultrasound

SPIS: Sono-pulmonary infection score
SPIS-mic: Sono-pulmonary infection score-microscopy SPIS-cult: Sono-pulmonary infection score-culture VAP: Ventilator-associated pneumonia

\section{Declarations}

This study has been approved by the institutional ethics committee of Sanjay Gandhi Postgraduate Institute of medical sciences (201531-DM-EXP).

Informed written consent was obtained from close relatives of the patient.

\section{Consent for publication: not applicable.}

All data and materials analyzed during the current study are available from the corresponding author on reasonable request from the critical care medicine department of Sanjay Gandhi Postgraduate Institute of Medical Sciences, Lucknow.

\section{Author Contributions}

SS contributed to the hypothesis, design, outline of the data, data collections, and drafting of the manuscript. AA contributed to data analysis, interpretation, review, and editing of the manuscript. $\mathrm{RP}$ contributed to the review and editing of the manuscript. AKB contributed to the interpretation of results. MG contributed to the interpretation of results and analysis of data. BP contributed to the interpretation and analysis of data. ZN contributed to the lung ultrasound and interpretation of the result.

\section{ACKNOWLEDGEMENT}

We gratefully acknowledge the help and support of the entire Critical Care Medicine staff.

\section{OrCID}

Sukhen Samanta @ https://orcid.org/0000-0002-3041-9529 Rupali Patnaik @ https://orcid.org/0000-0003-0104-7102

Afzal Azim (1) https://orcid.org/0000-0003-3077-5424

Mohan Gurjar (1) https://orcid.org/0000-0002-8489-0324

Arvind K Baronia @ https://orcid.org/0000-0002-2799-0708

Banani Poddar (1) https://orcid.org/0000-0002-1843-3037

Ratender K Singh (1) https://orcid.org/0000-0002-0811-5254

Zafar Neyaz @ https://orcid.org/0000-0002-5188-5211

\section{References}

1. Vincent JL, Bihari DJ, Suter PM, Bruining HA, White J, Nicolas-Chanoin $\mathrm{MH}$, et al. The prevalence of nosocomial infection in intensive care units in Europe. Results of the European Prevalence of Infection in Intensive Care (EPIC) Study. EPIC International Advisory Committee. JAMA 1995;274(8):639-644.

2. Heyland DK, Cook DJ, Griffith L, Keenan SP, Brun-Buisson C. The attributable morbidity and mortality of ventilator-associated pneumonia in the critically ill patient. The Canadian Critical Trials Group. Am J Respir Crit Care Med 1999;159(4 Pt 1):1249-1256. DOI: 10.1164/ajrccm.159.4.9807050.

3. Fartoukh M, Maitre B, Honoré S, Cerf C, Zahar JR, Brun-Buisson C. Diagnosing pneumonia during mechanical ventilation: the clinical pulmonary infection score revisited. Am J Respir Crit Care Med 2003;168(2):173-179. DOI: 10.1164/rccm.200212-1449OC.

4. Sperandeo M, Carnevale V, Muscarella S, Sperandeo G, Varriale A, Filabozzi P, et al. Clinical application of transthoracic ultrasonography in inpatients with pneumonia. Eur J Clin Invest. 2011;41(1):1-7. DOI: 10.1111/j.1365-2362.2010.02367.x. 
5. Lichtenstein D, Lascols N, Mezière G, Gepner A. Ultrasound diagnosis of alveolar consolidation in the critically ill. Intensive Care Med 2004;30(2):276-281. DOI: 10.1007/s00134-003-2075-6.

6. Zagli G, Cozzolino M, TerreniA, Biagioli T, Caldini AL, Peris A. Diagnosis of ventilator-associated pneumonia: a pilot, exploratory analysis of a new core based on procalcitonin and chest echography. Chest 2014;146(6):1578-1585. DOI: 10.1378/chest.13-2922.

7. Mongodi S, Via G, Girard M, Rouquette I, Misset B, Braschi A, et al. Lung ultrasound for early diagnosis of ventilator-associated pneumonia. Chest 2016;149(4):969-980. DOI: 10.1016/j.chest.2015.12.012.

8. Pugin J, Auckenthaler R, Milli N, Janssens JP, Lew PD, Suter PM. Diagnosis of ventilator-associated pneumonia by bacteriologic analysis of bronchoscopic and nonbronchoscopic "blind" bronchoalveolar lavage fluid. Am Rev Respir Dis 1991;143(5 Pt 1):1121-1129. DOI: $10.1164 /$ ajrccm/143.5_Pt_1.1121.

9. Croce MA, Swanson JM, Magnotti LJ, Claridge JA, Weinberg JA, Wood GC, et al. The futility of the clinical pulmonary infection scores in trauma patients. J Trauma 2006;60(3):523-528. DOI: 10.1097/01. ta.0000204033.78125.1b.

10. Schurink CAM, Van Nieuwenhoven CA, Jacobs JA, Rozenberg-Arska $M$, Joore HCA, Buskens $E$, et al. Clinical pulmonary infection score for ventilator-associated pneumonia: accuracy and inter-observer variability. Intensive Care Med 2004;30(2):217-224. DOI: 10.1007/ s00134-003-2018-2.

11. Fàbregas N, Ewig S, Torres A, El-Ebiary M, Ramirez J, de La Bellacasa JP, et al. Clinical diagnosis of ventilator associated pneumonia revisited: comparative validation using immediate post-mortem lung biopsies. Thorax 1999;54(10):867-873. DOI: 10.1136/thx.54.10.867.

12. Fartoukh M, Maitre B, Honoré S, Cerf C, Zahar JR, Brun-Buisson C. Diagnosing pneumonia during mechanical ventilation: the clinical pulmonary infection score revisited. Am J Respir Crit Care Med 2003;168(2):173-179. DOI: 10.1164/rccm.200212-1449OC.

13. Singh N, Rogers P, Atwood CW, Wagener MM, Yu VL. Short-course empiric antibiotic therapy for patients with pulmonary infiltrates in the intensive care unit. A proposed solution for an indiscriminate antibiotic prescription. Am J Respir Crit Care Med 2000;162(2):505511. DOI: 10.1164/ajrccm.162.2.9909095.

14. Bouhemad B, Zhang M, Lu Q, Rouby JJ. Clinical review: bedside lung ultrasound in critical care practice. Crit Care 2007;11(1):205. DOI: doi: $10.1186 / \mathrm{cc} 5668$.
15. Winer-Muram HT, Steiner RM, Gurney JW, Shah R, Jennings SG, Arheart KL, et al. Ventilator-associated pneumonia in patients with adult respiratory distress syndrome: CT evaluation. Radiology 1998;208(1):193-199. DOI: 10.1148/radiology.208.1. 9646813.

16. Bourcier JE, Paquet J, Seinger M, Gallard E, Redonnet JP, Cheddadi F, et al. Performance comparison of lung ultrasound and chest $x$-ray for the diagnosis of pneumonia in the ED. Am J Emerg Med 2014;32(2):115-118. DOI: 10.1016/j.ajem.2013.10.003.

17. Hu QJ, Shen YC, Jia LQ, Guo SJ, Long HY, Pang CS, et al. Diagnostic performance of lung ultrasound in the diagnosis of pneumonia: a bivariate meta-analysis. Int J Clin Exp Med 2014;7(1):115-121.

18. Inglis AJ, Nalos M, Sue KH, Hruby J, Campbell DM, Braham RM, et al. Bedside lung ultrasound, mobile radiography, and physical examination: a comparative analysis of diagnostic tools in the critically ill. Crit Care Resusc 2016;18(2):124.

19. Parlamento S, Copetti R, Di Bartolomeo S. Evaluation of lung ultrasound for the diagnosis of pneumonia in the ED. Am J Emerg Med 2009;27(4):379-384. DOI: 10.1016/j.ajem.2008.03.009.

20. Zanobetti M, Poggioni C, Pini R. Can chest ultrasonography replace standard chest radiography for evaluation of acute dyspnea in the ED? Chest 2011;139(5):1140-1147. DOI: 10.1378/chest.10-0435.

21. Reissig A, Copetti R, Mathis G, Mempel C, Schuler A, Zechner P, et al. Lung ultrasound in the diagnosis and follow-up of communityacquired pneumonia: a prospective, multicenter, diagnostic accuracy study. Chest 2012;142(4):965-972. DOI: 10.1378/chest.120364.

22. American Thoracic Society; Infectious Diseases Society of America. Guidelines for the management of adults with hospital-acquired, ventilator-associated, and healthcare-associated pneumonia. Am J Respir Crit Care Med 2005;171(4):388-416. DOI: 10.1164/rccm.200405644ST.

23. Hindler JF, Stelling J. Analysis and presentation of cumulative antibiograms: a new consensus guideline from the Clinical and Laboratory Standards Institute. Clin Infect Dis 2007;44(6): 867-873. DOI: $10.1086 / 511864$.

24. Becher RD, Hoth JJ, Rebo JJ, Kendall JL, Miller PR. Locally derived versus guideline-based approach to treatment of hospital-acquired pneumonia in the trauma intensive care unit. Surg Infect (Larchmt) 2012;13(6):352-359. DOI: 10.1089/sur.2011.056. 